\title{
El paciente polimedicado
}

\author{
The polymedicated patient
}

\author{
Fernando Diz-Lois Martínez¹, Ovidio Fernández Alvarez², Ceferino Pérez Vázquez ${ }^{3}$ \\ ${ }^{1}$ Médico internista emérito, CHUAC \\ ${ }^{2}$ Médico internista emérito $\mathrm{CHOU}$ \\ ${ }^{3}$ Médico de Familia, Xerencia de Atención Primaria. A Coruña
}

\section{Introducción}

La polimedicación o polifarmacia se define como: la administración de varios medicamentos durante un tiempo prolongado. Para algunos consiste en la toma de 50 más medicamentos durante al menos seis meses, para otros entre 5 a 10 medicamentos, siendo necesario consignar también remedios de herbolario, al menos algunos con actividad biológica significativa (sedantes, laxantes, excitantes, anticolinérgicos, anabolizantes etc. $)^{1}$. Pero más que una cifra concreta, en la práctica, interesan criterios cualitativos de adecuación terapéutica. De todas maneras a partir de de 6 medicamento tomados de forma crónica existe una asociación progresiva con inadecuación de la prescripción?2.

La polimedicación es particularmente importante en gente mayor, que con frecuencia sufre pluripatología. En USA un $20 \%$ de beneficiarios de medicare tienen 6 o más procesos crónicos y el $50 \%$ reciben 50 más medicamentos ${ }^{3}$. Es necesario, sin embargo diferenciar la pluripatologia de los "achaques de la edad" como: algias temporales, disminución de las capacidades sensoriales, pérdida de memoria, pérdida de agilidad y fuerza, aumento de peso, estreñimiento, tendencias depresivas, insomnio etc, muchos de los cuales no se remedian a medio plazo con medicamentos, sino con apoyos familiares o sociales y promoción de ciertas precauciones y actividades adecuadas en la vida diaria de la persona. No se deben sustituir los cuidados y afectos que la gente mayor necesita por píldoras.

En España la frecuencia de polifarmacia en mayores de 65 años no institucionalizados es de 2,6 \pm 2 medicamento por paciente ${ }^{4}$. La frecuencia de polimedicados aumenta en institucionalizados, en relación a los comunitarios ${ }^{5}$, también en presencia de demencia ${ }^{6}, 0$ cuando el paciente tiene problemas de movilidad. Los medicamentos utilizados con mayor frecuencia son, antihipertensivos, ansiolíticos, vasodilatadores, hipnóticos, anti-inflamatorios, digoxina y espasmolíticos ${ }^{5,7}$. La automedicación es otro factor responsable de la polifarmacia, afecta en nuestro medio a cerca de un tercio de los pacientes mayores de 75 años ${ }^{4}$.

El promedio de ingestión de fármacos se mantiene hasta cerca de los 90 años (alrededor de 8 en el medio comunitario), después declina ligeramente ${ }^{8}$.
Es necesario diferenciar lo que se receta de la toma real, por parte del enfermo, de la medicación prescrita. Es lógico pensar que un paciente no sea cumplidor (a menos que tenga una enfermedad grave) si tiene un plan terapéutico con más de seis medicamentos, cuando el grado de cumplimiento medio con uno o dos fármacos es menor de un $70 \%$. El incumplimiento terapéutico afecta al 30-50\% de los ancianos y en los polimedicados alcanza el $47,6 \% 12^{9}$.

Por eso es importante distinguir lo que es la poli-prescripción de lo que es la poli-terapia (entendiendo por esta última lo que realmente toma el enfermo). Aquella tiene importancia en lo que se refiere a gasto farmacéutico y esta es importante a la hora de evaluar la eficacia y efectos tóxicos de los medicamentos ${ }^{10}$.

La polimedicación aunque, como expresión vulgar, tiene una connotación negativa a veces es necesaria. Administrar incluso más de 6 principios activos a un mismo paciente es imprescindible cuando sus múltiples procesos así lo requieren o sí, como ocurre con enfermedades graves o potencialmente graves, necesitan de varios medicamentos para su control.

\section{Polifarmacia y toxicidad medicamentosa}

Es difícil de justificar la necesidad de tomar más de 6-8 principios activos de forma crónica. Esto en sí mismo constituye una nueva enfermedad, ya que el riesgo de provocar efectos tóxicos importantes con tal combinación es elevado. Amplios estudios poblacionales han demostrado que la ocurrencia de reacciones adversas medicamentosas es 50 por mil personas y año, de las cuales el $0,7 \%$ son mortales, el $8,9 \%$ son potencialmente mortales, el $28,3 \%$ son graves y el $62 \%$ son significativas ${ }^{11}$. El uso de 60 más fármacos es un predictor de mortalidad independiente (OR:3) ${ }^{12}$. El riesgo lógicamente es proporcional al número de medicamentos tomados regularmente ${ }^{13}$.

En nuestro medio alrededor de un tercio de la población estudiada presenta problemas potenciales derivados del consumo de fármacos, por interacciones y/o uso inapropiado. Las variables asociadas son: un mal estado de salud percibido y el mayor consumo de fármacos ${ }^{14}$. Es un problema grave tanto en el hospital como demostró el estudio nacional sobre efectos adversos ligados a hospitalización ${ }^{15}$, como en la comunidad 
quedando claro en el estudio APEAS (estudio sobre la seguridad de los pacientes en atención primaria de salud), en el que se observó que el 48,2\% de los efectos adversos ligados a la asistencia sanitaria están causados por la medicación ${ }^{16}$. Algunas observaciones han detectado efectos adversos en cerca del $100 \%$ de los pacientes cuando ingieren más de 10 medicamentos regularmente ${ }^{17}$, aunque habría que diferenciar lo que son efectos colaterales esperados de verdaderas reacciones adversas.

Existen datos que sugieren que después de controlar otras morbilidades la polimedicación se asocia a un deterioro físico y mental ${ }^{18}$ y particularmente fractura de fémur asociada a caídas casuales ${ }^{19}$.

Un hecho muy conocido son las cascadas de prescripción originadas por los efectos colaterales de la medicación interpretados a veces como una nueva enfermedad ${ }^{20}$. Como el parkinson inducido por antipsicóticos o metoclopramida², o la diarrea e incontinencia urinaria inducido por inhibidores de la colinesterasa (donecepilo, rivastigmina etc) ${ }^{22}$.

Las guías de práctica clínica pueden inducir a polimedicar cuando el paciente tiene varios procesos ${ }^{3,23}$. Es fácil comprender que un paciente diabético con EPOC, cardiopatía isquémica y osteoartritis necesite más de 10 medicamentos. En estos casos es esencial juzgar lo que es realmente importante para el paciente en relación a su tipo de vida y pronóstico.

Así pues hay que tener en cuenta el balance riesgo beneficio de un medicamento indicado en una situación determinada. A veces es necesario sacrificar su máxima eficacia en un problema determinado para evitar complicaciones (ej. sangrado digestivo en tratamiento con dicumarínicos en la FA, en cuyo caso 0 se suspende 0 se mantiene con un INR subóptimo, o suspender o minimizar la dosificacion de benzodiazepinas para evitar caidas) $)^{24}$ etc.

Como ley general de la naturaleza la polimedicacion como fenómeno sigue el principio de Wilfredo Pareto: "una minoría de causas es responsable de una mayoría de efectos". Una minoría consume una mayoría de recursos. En nuestro caso esa minoría se refiere al escaso $20 \%$ de pesonas mayores de 65 años. En España el 17\% de la poblacion tiene mas de 65 años y consumen el $73 \%$ del gasto farmacéutico ${ }^{17}$. Siempre ocurrirá que las personas mayores consuman más medicación. De lo que se trata es evitar que una misma persona consuma medicación que no sea imprescindible a su condición. Todos estamos de acuerdo en que se prescribe demasiado.

Una serie de factores que se suman y favorecen la polimedicación ${ }^{25}$.

Por parte del médico: En primer lugar la fragmentación de la asistencia. Cuando hay varios médicos prescribiendo al mismo paciente aumenta el número de fármacos que el paciete toma y el riego de efectos secundarios ${ }^{26}$. Hay un exceso de protagonismo de los especialistas en lo que debería ser atención primaria. El médico generalista debería ser quien llevara la dirección de la asistencia al paciente y así evitar consultas innecesarias y que las distintas prescripciones por distintos especialistas se fueran sumando sin un control adecuado. Suele acaecer que hay una inhibición por parte de los especialistas en revisar la medicación prescrita por otros médicos ajenos a su especialidad, prueba de ello es que muchas veces en los informes de alta de hospital o de consulta, al escribir el tratamiento, el especialista (también el internista) hace el listado de los medicamentos relacionados con la causa de la consulta y le se añade "resto igual", y en este "resto igual" puede estar la clave del diagnóstico o de la causa de la descompensación. Otras circunstancias que pueden inducir al médico a recetar son: la fugacidaz en la relación con el enfermo (es más fácil recetar que historiar) y la colaboración con la promoción de fármacos comerciales. Por parte del paciente, la pluripatología, la pèrdida progresiva de autocuidados, el estilo de vida poco saludable, la gratuidad de la asistencia y de la medicación.

En relación con la industria farmacéutica, la promoción a veces excesiva de sus productos buscando la colaboración del médico, o una investigación dirigida a fármacos clónicos de otros que han tenido éxito comercial favorecen la polifarmacia. Hay que reconocer sin embargo que en muchos casos una parte muy significativa del armamentario terapéutico es novedosa y posterior a los estudios de la carrera de medicina del médico, por lo que la información que tiene este de una parte importante de medicamentos es a partir de la industria farmaceútica y de estudios financiados por la misma, esto puede ser causa de un sesgo a la hora de recetar, sin quitarle importancia a la labor de educación médica propiciada por la industria incluída la formación continuada reglada y su colaboración en los congresos de medicina ${ }^{27}$.

Los medios de comunicación también influyen con una excesiva y mal entendida publicidad así como la promoción involuntaria de falsas expectativas. La administración peca por exceso en su aprobación de principios activos similares así como productos genéricos.

Cuando todos los agentes protagonistas de la asistencia sanitaria están orientados en la misma dirección, hacia la polifarmacia, es difícil cambiar esta tendencia. Y tal como está organizada la asistencia sanitaria, tanto el médico, como el farmacéutico, la industria y el paciente siguen la misma ruta la del consumo. A esto se añade que la sociedad moderna que se autodefine como sociedad del bienestar, favorece, incluso induce, al consumo de bienes y servicios. La búsqueda de la satisfacción personal por el placer como un fin 
en sí mismo, en vez de ser consecuencia de un esfuerzo previo, tiene como uno de sus resultados la sustitución de estilos de vida saludables por la toma de pastillas. Es más fácil tomar medicamentos etc. para evitar la osteoporosis 0 el sobrepeso que practicar una actividad física regular.

\section{Control y detección del paciente polimedicado}

El diagnóstico de la polimedicación es obvio, aunque quizás no tanto si tenemos en cuenta que el paciente no siempre toma lo que está escrito, además se automedica o toma productos activos de herboristería. Es por lo tanto necesario realizar una anamnesis adecuada, a veces laboriosa. Siempre se hace necesario en la consulta valorar si la medicación que está tomando el enfermo es: necesaria, potencialmente perjudicial, interacciona o está provocando efectos secundarios importantes. La valoración del clínico en la evitación de una politerapia inadecuada es lo más importante ya que es él quien conoce las peculiaridades del paciente.

Se han desarrollado criterios externos que se utilizan para valorar la adecuación de la prescripción no solo en sentido de exceso sino también por defecto. En la aplicación de criterios objetivos es necesario tener en cuenta no solo la propia indicación sino factores psíquicos, familiares y sociales ${ }^{25}$. Beers y colaboradores, en 1992, describieron 30 factores para aplicar en la clínica y determinar la calidad de la prescripcion de medicación utilizada de forma habitual ${ }^{28}$. Estos criterios fueron actualizados en el año 2003, incrementando además a 48 medicamentos o clases de medicamentos potencialmente contraindicados en pacientes mayores ${ }^{29}$. Estos criterios son ampliamente utilizados, y mediante su aplicación se ha detectado un 20-30\% de prescripción inadecuada sobre todo en ancianos asilados, siendo esto responsable de una morbilidad significativa $30,31,32$.

Estos criterios no parecen adecuados para países europeos ya que los formularios son distintos y van cambiando, las actitudes prescriptoras también son diferentes así como las exigencias de la población y la idiosincrasia social. En un estudio de consenso realizado por Gallagher y colaboradores en Irlanda, se pactaron una sere de criterios más amplios que los de Beers, actualizados y adaptados a la farmacopea europea, que son más sensibles y específicos para detectar errores de prescripción sobre todo en ancianos ${ }^{33}$. Hay una excelente versión adaptada a la farmacopea y tipo de asistencia de España ${ }^{34}$.

Existen otras versiones con criterios diferentes tratando de simplificar su utilización para uso asistencial, como la canadiense. Ellos establecen una clasificación de medicamentos en tres categorías según su posible morbilidad: las contraindicadas en ancianos, las que producen interacciones con la enfermedad, 0 interacciones medicamentosas ${ }^{35}$.
Otro modo de hacerlo es centrarse en medicamentos especialmente peligrosos en ancianos por la frecuencia de su utilización y su especial peligrosidad, como son los los sedantes y los que tienen efecto anticolinérgico, como es el índice de "sobrecarga de medicamentos" o "drug burden index" expresada matemáticamente con una fórmula ${ }^{36}$.

El Medication Appropriateness Index (MAl) $)^{37}$ mide la adecuación de cada uno de los medicamentos del paciente a través de 10 ítems. Tiene el inconveniente de que su aplicación en la consulta puede llevar tiempo al tener que elaborar un indice global resultado de una puntuación por cada uno de los 10 items.

Todos estos sistemas de medir la adecuación de la prescripción han sido resumidas de forma extensa en una tesis doctoral38.

Lo importante en la práctica de todos estos sistemas de control explícitos es no solo su grado de precisión para captar errores de prescripción, sino también su fácil aplicación por el médico en la clínica diaria. En nuestro país, el método más utilizado es el de detección de los Resultados Negativos de la Medicación (RNM) ${ }^{39}$, que se definen como los resultados en la salud del paciente no adecuados al objetivo de la farmacoterapia y asociados al uso de medicamentos.

\section{Métodos de intervención para adecuar la prescripción}

\section{Estructurales:}

Dar más protagonismo al médico general en la asistencia, evitando que el especialista haga el trabajo propio del médico generalista ${ }^{40}$. El médico de cabecera debe de ser el reponsable final del paciente y es quien tiene que ordenar el plan terapéutico, contando naturalmente con el especialista. Es necesario mejorar la relacion entre redes asistenciales, la historia clínica común entre la medicina hospitalaria, ambulatoria, servicios de urgencia y asistencia primaria es una herramienta fundamental para conseguir la información necesaria para poner orden en la medicación de cada paciente. Pero actualmente existe una desnivelación asistencial a favor de las especialidades que es necesario compensar con un progresivo desarrollo de la Atención Primaria.

En experiencias previas en otros países con formularios estratificados y el copago han tenido como consecuencia disminución del uso de medicamentos ${ }^{41}$. Pero es necesario en caso del copago tomar medidas para evitar la no utilización de medicación necesaria. La semFYC está en desacuerdo con la aplicación de copagos en los servicios sanitarios y en caso de aplicarlos debería hacerse como estudio piloto experimental controlando múltiples variables y observar resultados tanto económicos como clínicos. Y en lo que se refiere a copago en farmacia replantearse el sistema actual hacia modelos más equitativos. ${ }^{42}$ 
En cuanto a utilización de formularios, en Galicia el catálogo de medicamentos ha disminuido el gasto aunque la prescripción por parte de los médicos se mantiene alta.

\section{Organizativos:}

Son de tres tipos,

1. Educacionales dirigidos a pacientes, a asilados, 0 a médi$\cos ^{43,44}$.

2. Llamadas atención a prescriptores de forma automática a traves de la red ${ }^{45,46}$.

3. Intervención del farmacéutico en distintos puntos de la prescripción ${ }^{9,47,48}$.

Todos han sido eficaces al menos a corto o medio plazo para reducir el número de medicamentos, algunos también para mejorar la calidad de la prescripción. La mayoría no han sido diseñados para valorar el factor coste/beneficio pero muchos sí han demostrado una disminución de efectos adversos ${ }^{49,50,51}$. En nuestra comunidad hay 110.000 pacientes que toman mas de 6 medicamentos cada día y 15.000 en los se han prescrito más de 15 medicamentos. La Dirección de Asistencia Sanitaria de la Consellería de Sanidade ha puesto en marcha un programa de mejora de tratamiento de enfermos crónicos "mediante el asesoramiento sobre el uso de fármacos, educación sanitaria y una ajustada colaboración entre los profesionales sanitarios", con los objetivos de: "detectar y resolver problemas relacionados con el uso de medicamentos, mejorar el cumplimiento terapéutico de los pacientes crónicos polimedicados y mejorar el conocimiento de los pacientes respecto del uso e indicaciones de los medicamentos". El proceso se inicia con una alerta por parte de la "Unidad de apoyo a la Prescripción" y a través del farmacéutico del centro de salud se hace una propuesta de intervención, tomando la decisión final el médico o en su caso la enfermera en una consulta dirigida al enfermo crónico.

Es indudable que cualquier método que recuerde al prescriptor la necesidad de racionalizar la terapia es bueno para mejorar la calidad del tratamiento y disminuir los efectos secundarios, pero nada puede sustituir una buena anamnesis para poder prescribir bien.

\section{Bibliografia}

1. Ferner RE, Aronson JK. Communicating information about drug safety. BMJ 2006 333:143-145.

2. Fialová D, Topinková E, Gambassi G, Finne-Soveri H, Jonsson PV, Carpenter I, Schroll $\mathrm{M}$ et al. Potentially inappropriate medication use among elderly home care patients in Europe. JAMA 2005:293:1348-1358.

3. Tinetti ME, Bogardus ST Jr, Agostini JV. Potential pitfalls of disease-specific guidelines for patients with multiple conditions. N Engl J Med 2004; 351:2870-4.

4. Valderrama Gama E, Rodríguez Artalejo F, Palacios Díaz A, Gabarre Orús P, Pérez del Molino Martín J. Consumo de medicamentos en los ancianos: resultados de un estudio poblacional. Rev Esp Salud Pública. 1998;72:209-19.

5. Sociedad Española de Geriatría y Gerontología. Guías de actuación clínica. Polifarmacia. 2000 citado por Comité Editorial de las Áreas 1, 2, 3 y 7 de Atención Primaria. Servicio Madrileño de Salud. Utilización de medicamentos en el anciano. Notas Farmacoter. 2008:15:29-36.

6. Planas-Pujol X, López-Pousa S, Vilalta-Franch J, Monserrat-Vila S, Garre-Olmo J. Perfil de consumo farmacológico y función cognoscitiva en edad avanzada: estudio de población general no institucionalizada. Neurología.2010; 25:498-506.

7. Gavilán Moral E, Morales Suárez-Varela MT, Hoyos Esteban JA, Pérez Suanes AM. Polimedicación y prescripción de fármacos en pacientes ancianos inmovilizados que viven en la comunidad. Aten Primaria. 2006;38:476-80.

8. Escamilla Fresnadillo JA, Castañer Niño 0, Benito López S, Ruiz Gil E, Burrull Gimeno M, Sáenz Moya N. Motivos de incumplimiento terapéutico en pacientes mayores polimedicados, un estudio mediante grupos focales. Aten Primaria. 2008:40:81-85.

9. Velasco Sánchez V, Juárez Molera M, López Parra E, Salamanca Sanz Al, Barrios Martos E y Santos Álvarez P. Análisis de las interacciones medicamentosas en población extrahospitalaria mayor de 65 a ños. Semergen. 2011;37:233-7.

10. Rollason V, Vot N. Reduction of polypharmacy in the elderly. A systematic review of the role of the pharmacist. Drugs Aging 2003;20:817-832.

11. Gurwitz JH, Field TS, Harrold LR, Rothschild J, Debellis K, Seger AC. Incidence and Preventability of Adverse Drug Events Among Older Persons in the Ambulatory Setting. JAMA. 2003; 289:1107-16.

12. Incalzi AR, Capparella O, Gemma A, Porcedda P, Raccis G, Sommella L. A simple method of recognizing geriatric patients at risk for death and disability. J. Am Geriatr Soc. 1997; 45:945-8.

13. Risk factors for adverse drug events among nursing home residents. Field TS, Gurwitz JH, Avorn J, McCormick D, Jain S, Eckler M, Benser M, Bates DW Arch Intern Med. 2001;161:1629-1634

14. Arbás E, Garzón R, Suárez A, Buelga C, Pozo M, Comas A, et al. Relación entre nivel de el consumo y toxicidad. Consumo de medicamentos en mayores de 65 años: problemas potenciales y factores asociados. Aten Primaria. 1998;23:165-170.

15. Estudio Nacional sobre los Efectos Adversos ligados a la Hospitalización. ENEAS 2005. Plan de calidad del sistema nacional de salud. Informe. Febrero 2006

16. Estudio APEAS. Estudio sobre la seguridad de los pacientes en atención primaria de salud. Madrid: Plan de calidad, Ministerio de Sanidad y Consumo, 2008

17. Cmité Editorial de las Áreas 1, 2, 3 y 7 de Atención Primaria. Servicio Madrileño de Salud. Utilización de medicamentos en el anciano. Notas Farmacoter. 2008;15:29-

18. Magaziner J, Cadigan DA, Fedder DO, Hebel JR. Medi- cation use and functiona decline among community dwelling older women. J Aging Health. 1989;1:470- 484

19. Lai SW, Liao KF, Liao CC, Muo CH, Liu CS, Sung FC. Polypharmacy correlates with increased risk for hip fracture in the elderly: a population-based study. Medicine (Baltimore). 2010;89:295-9.

20. Rochon PA, Gurwitz JH. Optimising drug treatment for elderly people: the prescribing cascade. BMJ 1997; 315:1096-9.

21. Rochon PA, Stukel TA, Sykora K, Gill S, Garfinkel S, Anderson GM, Normand SL, Mamdani M, Lee PE, Li P, Bronskill SE, Marras C, Gurwitz JH Atypical antipsychotics and parkinsonism. Arch Intern Med. 2005;165(16):1882-8.

22. Gill SS, Mamdani M, Naglie G, Streiner DL, Bronskill SE, Kopp A, Shulman KI, Lee PE, Rochon PA. A prescribing cascade involving cholinesterase inhibitors and anticholinergic drugs. Arch Intern Med. 2005;165:808-13.

23. Boyd CM, Darer J, Boult C, Fried LP, Boult L, Wu AW. Clinical practice guidelines and quality of care for older patients with multiple comorbid diseases: implications for pay for performance. JAMA. 2005;294:716-724

24. Steinman MA, Hanlon JT. Managing medications in clinically complex elders: "There's got to be a happy medium." JAMA 2010; 304:1592-1601.

25. Villafaina Barroso A, Gavilan Moral E. Pacientes polimedicados frágiles, un reto para el sistema sanitario. IT del Sistema Nacional de Salud. Volumen 35, № 4/2011.

26. Green JL, Hawley JN, Rask KJ. Is the number of prescribing physicians an independent risk factor for adverse drug events in an elderly outpatient population? Am J Geriatr Pharmacother. 2007;5:31-9.

27. Pérez Vázquez C, Serrano Peña J, Louro González A. Plan de incentivación a la racionalización de la prescripción en receta médica oficial en Atención Primaria (PIRPRMOAP). Procedimiento de actuación. Cad Aten Primaria. 2009:16:81-85. 
28. Beers MH, Ouslander JG, Rollingher I, Reuben DB, Brooks J, Beck JC. Explicit criteria for determining inappropriate medication use in nursing home residents. UCLA Division of Geriatric Medicine. Arch Intern Med. 1991;151(9):1825.

29. Fick DM, Cooper JW, Wade WE, Waller JL, Maclean JR, Beers MH. Arch Intern Med. 2003:163(22):2716.

30. 30.- Willcox SM, Himmelstein DU, Woolhandler S. Inappropriate drug prescribing for the community-dwelling elderly. JAMA. 1994;272:292-6.

31. Lau DT, Kasper JD, Potter DE, Lyles A, Bennett RG. Hospitalization and death associated with potentially inappropriate medication prescriptions among elderly nursing home residents. Arch Intern Med. 2005;165:68-74.

32. Jano E, Aparasu RR. Healthcare outcomes associated with beers' criteria: a systematic review. Ann Pharmacother. 2007;41: 438-447.

33. 33.- Gallagher P, Ryan C, Byrne S, Kennedy J, Oâ Mahony D. STOPP (Screening Tool of Older Persons Prescriptions) and START (Screening Tool to Alert doctors to Right Treatment). Consensus validation. Int J Clin Pharmacol Ther 2008; 46:72-83.

34. Delgado E, Muñoz M, Montero B, Sánchez C, Gallagher P, Cruz-Jentoft A.. Prescripción inapropiada de medicamentos en los pacientes mayores: Ios criterios STOPP/ START. Rev Esp Geriatr Gerontol 2009;44:273-9.

35. McLeod PJ, Huang AR, Tamblyn RM, Gayton DC. Defining inappropriate practices in prescribing for elderly people: a national consensus panel. CMAJ. 1997:156(3):38591.

36. Hilmer SN, Mager DE, Simonsick EM, Cao Y, Ling SM, Windham BG, Harris TB, HanIon JT, et al. Drug Burden Index to Define the Functional Burden of Medications in Older People. Arch Intern Med. 2007;167:781-787.

37. Hanlon JT, Schmader KE, Samsa GP, Weinberger M, Uttech KM, Lewis IK, Cohen $\mathrm{HJ}$, and Feussner JR.. A method for assessing drug therapy appropriateness. J Clin Epidemiol 1992; 45:1045-1051.

38. Spinewine A. Appropriate use of medicines in care of the elderly - factors underlying inappropriateness, and impact of the clinical pharmacist. Thèse présentée a Université Catholique de Louvain. 2006. http://www.farm.ucl.ac.be/cfcl/Theses/ Spinewine/Spinewine-thesis.pdf. Consultada el 28 Enero 2012.

39. Comité de Consenso GIAF-UGR, GIFAF-USE, GIF-UGR. Tercer Consenso de Granada sobre Problemas Relacionados con Medicamentos (PRM) y Resultados Negativos asociados a la Medicación (RNM). Ars Pharm 2007;48:5-17.

40. Norman G. Levinsky, M.D. Recruiting for primary care. N Engl J Med 1993; 328:656660.
41. Lu CY, Ross-Degnan D, Soumerai SB and Pearson SA. Interventions designed to improve the quality and efficiency of medication use in managed care: A critical review of the literature - 2001-2007. BMC Health Services Research 2008; 8:75-87.

42. COPAGO: conclusiones desde la evidencia científica. Documento semFYC, octubre de 2010. Editorial. Aten Primaria. 2011:43:1-2

43. Williams ME, Pulliam CC, Hunter R, Johnson TM, Owens JE, Kincaid J, et al. The short-term effect of interdisciplinary medication review on function and cost in ambulatory elderly people. J Am Geriatr Soc. 2004;52:93-8.

44. Fillit HM, Futterman R, Orland BI, Chim T, Susnow L, Picariello G, et al. Polipharmacy management in Medicare managed care: changes in prescribing by primary care physicians resulting from a program promoting medications reviews. Am J Manag Care. 1999;5:587-94.

45. Monane M, Matthias DM, Nagle BA, Kelly MA. Improving prescribing patterns for the elderly through an online drug utilization review intervention: a system linking the physician, pharmacist, and computer. JAMA. 1998;280:1249-52.

46. Raebel MA, Charles J, Dugan J, Carroll NM, Korner EJ, Brand DW, et al. Randomized trial to improve prescribing safety in ambulatory elderly patients. J Am Geriatr Soc. 2007:55:977-85.

47. Castelino RL, Bajorek BV, and Chen TF. Targeting Suboptimal Prescribing in the Elderly: A Review of the Impact of Pharmacy Services. Ann Pharmacother June 2009 43:1096-1106.

48. Zermansky AG, Alldred DP, Petty DR, David K. Raynor DK, Freemantle N, Eastaugh $\mathrm{J}$ and Bowie P. Clinical medication review by a pharmacist of elderly people living in care homes, randomised controlled trial. Age and Ageing. 2006; 35: 586-591.

49. Obtaining a thorough medication history is very important before any new medication is prescribed. Hajjar ER, Cafiero AC, Hanlon JT. Polypharmacy in elderly patiens. Am J Geriatr Pharmacother. 2007:5:345-351.

50. Forsetlund L, Eike MC, Gjerberg E, and Vist GE. Effect of interventions to reduce potentially use of drugs in nursing homes: a systematic review of randomised controlleed trials. BMC Geriatr. BMC Geriatr. 2011; 11: 16-34.

51. George J, Elliott RA, Stewart DC. A systematic review of interventions to improve medication taking in elderly patients prescribed multiple medications. Drugs Aging. 2008;25:307-24 